\title{
Absolute polarization angle calibration using polarized diffuse Galactic emission observed by BICEP
}

\author{
Tomotake Matsumura $^{a}$, Peter Ade ${ }^{b}$, Denis Barkats ${ }^{c}$, Darcy Barron ${ }^{d}$, John O. Battle ${ }^{e}$, Evan M. \\ Bierman $^{e}$, James J. Bock ${ }^{e, a}$, H. Cynthia Chiang $f$, Brendan P. Crill ${ }^{e, a}$, C. Darren Dowell ${ }^{e, a}$, \\ Lionel Duband $^{g}$, Eric F. Hivon ${ }^{h}$, William L. Holzapfel ${ }^{i}$, Viktor V. Hristov ${ }^{a}$, William C. Jones ${ }^{f}$, \\ Brian G. Keating ${ }^{e}$, John M. Kovac ${ }^{j}$, Chao-Lin Kuo ${ }^{k}$, Andrew E. Lange ${ }^{e, a}$, Erik M. Leitch ${ }^{e}$, \\ Peter V. Mason ${ }^{a}$, Hien T. Nguyen ${ }^{e}$, Nicolas Ponthieu ${ }^{l}$, Clem Pryke ${ }^{m}$, Steffen Richter ${ }^{a}$, \\ Graca M. Rocha ${ }^{e}$, Yuki D. Takahashi ${ }^{i}$, Ki Won Yoon ${ }^{n}$. \\ ${ }^{a}$ California Institute of Technology, Pasadena, USA; ${ }^{b}$ University of Wales, Cardiff, CF243YB, \\ Wales, UK; ${ }^{c}$ Joint ALMA Office, Chile; ${ }^{d}$ University of California, San Diego, USA; ${ }^{e}$ Jet

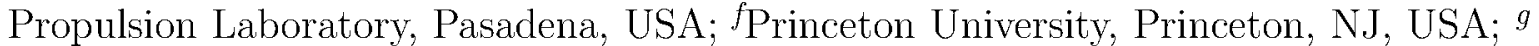 \\ Commissariat à l'Énergie Atomique, Grenoble, France; ${ }^{h}$ Institut d'Astrophysique de Paris, \\ France; ${ }^{i}$ University of California, Berkeley, USA; ${ }^{j}$ Harvard University, USA; ${ }^{k}$ Stanford \\ University, Palo Alto, USA; ${ }^{l}$ Universite Paris XI, Orsay, France; ${ }^{m}$ University of Chicago, USA; \\ ${ }^{n}$ National Institute of Standards and Technology, Boulder, USA.
}

\begin{abstract}
We present a method of cross-calibrating the polarization angle of a polarimeter using BICEP Galactic observations. BICEP was a ground based experiment using an array of 49 pairs of polarization sensitive bolometers observing from the geographic South Pole at 100 and $150 \mathrm{GHz}$. The BrCEP polarimeter is calibrated to \pm 0.01 in cross-polarization and less than $\pm 0.7^{\circ}$ in absolute polarization orientation. BICEP observed the temperature and polarization of the Galactic plane $\left(\right.$ R.A $=100^{\circ} \sim 270^{\circ}$ and Dec. $\left.=-67^{\circ} \sim-48^{\circ}\right)$. We show that the statistical error in the $100 \mathrm{GHz}$ BICEP Galaxy map can constrain the polarization angle offset of WMAP W band to $0.6^{\circ} \pm 1.4^{\circ}$. The expected $1 \sigma$ errors on the polarization angle cross-calibration for Planck or EPIC are $1.3^{\circ}$ and $0.3^{\circ}$ at 100 and $150 \mathrm{GHz}$, respectively. We also discuss the expected improvement of the BICEP Galactic field observations with forthcoming BICEP2 and KECK observations.
\end{abstract}

Keywords: cosmic microwave background polarization, millimeter wave, calibration source, polarized galactic emission, polarization calibration

\section{INTRODUCTION}

The polarization of the cosmic microwave background radiation (CMB) provides a tool for probing the physics of the early Universe. The CMB polarization field is decomposed into even parity E-mode and odd parity B-mode. ${ }^{1}$ Primordial density perturbations result in only E-mode polarization. The E-mode signal was discovered by DASI and characterized by experiments including BoOMERANG, CBI, MAXIPOL, QUAD, WMAP, and BICEP. ${ }^{2-8}$ Scientific interest in the CMB community moves toward detection of the B-mode signal, which originates from a primordial inflationary gravitational wave background and weak gravitational lensing. ${ }^{9}$ Numerous kilo-pixel array experiments, including EBEX, BICEP2, KECK, PolArBeAR, QUIET, and SPIDER, are in operation or under construction to search for B-mode polarization. ${ }^{10-13,15,16}$

While the sensitivity of an experiment increases by employing a large number of detectors, the requirements for controlling systematic effects becomes also stringent. ${ }^{17}$ Among systematic effects in the experiment, the polarization angle of the detectors is one of the most important quantities to be calibrated. Any miscalibration of the absolute polarization angle of a polarimeter mixes E-mode to B-mode signals, and therefore produces a false B-mode signal. Furthermore, such mixed E and B-mode signals are correlated and non-zero EB correlation indicates a false detection of the CPT violation or cosmic birefringence. ${ }^{18-20}$

(Send correspondence to T. Matsumura)

T. Matsumura: E-mail: tm@astro.caltech.edu, Telephone: +1 626-395-2147.

Millimeter, Submillimeter, and Far-Infrared Detectors and Instrumentation for Astronomy V, edited by Wayne S. Holland, Jonas Zmuidzinas, Proc. of SPIE Vol. 7741, 774120 (C) 2010 SPIE - CCC code: 0277-786X/10/\$18 - doi: 10.1117/12.856855 
The strategy for calibrating the absolute angle of a polarimeter may differ depending on the size of the telescope and the platform of the observatory, i.e. ground-based, balloon-borne and space-borne. The polarization angle of a small ground-based telescope like BICEP can be calibrated nearly end-to-end in the optical chain without replying on a calibration source on the sky but rather with a precisely oriented polarized source in front of the aperture in nominal observing conditions. On the other hand, large telescopes or any balloon- or space-borne telescopes are difficult to calibrate in nominal observing conditions without using a polarized sky signal.

Commonly used polarized sources at millimeter wavelengths in the sky are the Crab nebula (Tau A) and Centaurus A (Cen A). The Crab nebula is a supernova remnant that emits highly polarized radiation. Aumont et al. presented the intensity and polarized signals of the Crab nebula observed by IRAM at $90 \mathrm{GHz} .{ }^{21} \mathrm{Cen} A$ is an active galactic nucleus and Zemcov et al. reported the measurements of Cen A using the QUaD telescope. ${ }^{22}$ The reflection from the rim of the Moon is another source of the polarized calibration at millimeter wavelengths for a detector that has a large dynamic range. ${ }^{23}$ WMAP presented the measurements of the polarized celestial sources, including the Crab nebula, from 23 to $94 \mathrm{GHz} .{ }^{24}$ The measurements of the Crab nebula show a consistent polarization angle with Aumont et al.. Planck satellite is planning to use this source to calibrate the polarization angle of LFI and HFI detectors. ${ }^{25,26}$

While these highly polarized compact sources are widely used for a polarization calibration, a high signal-tonoise diffuse Galactic polarized signal observed by BICEP is another polarized source for the angle calibration on the sky. BICEP was a millimeter-wave bolometric polarimeter that is designed to observe the CMB polarization. ${ }^{27}$ BICEP employs a refractive telescope with a small $24 \mathrm{~cm}$ aperture, simplifying the characterization of the end-toend performance of the polarimeter's entire optical chain. While the observation of BrCEP is concentrated on the sky region that is minimally contaminated by the dust and synchrotron emissions, one-fifth of the observational time is dedicated to the Galactic plane observations. With systematic effects well controlled, a high signal-tonoise map of the diffuse polarized signal over the Galactic plane makes a standard calibration source on the sky for ongoing and forthcoming CMB polarization experiments.

In Section 2 we discuss the statistical and systematic uncertainties in the BICEP polarized Galaxy map. In Section 3 we discuss the formalism to cross-calibrate the polarization maps produced by an unknown absolute polarization angle polarimeter using the BICEP polarization map. In Section 4, we apply this recipe to crosscalibrate between the BICEP $100 \mathrm{GHz}$ and WMAP W band maps, as well as compute the expected constraint on the polarization offset angle for Planck and EPIC.

\section{BICEP POLARIZED GALAXY MAP}

BICEP was a ground based telescope observing from the geographic South Pole. The polarimeter consists of a two lens refractive telescope with a $24 \mathrm{~cm}$ aperture and 49 pairs of polarization sensitive bolometers (PSBs) at $100 \mathrm{GHz}$ and $150 \mathrm{GHz}$ with a corresponding beam widths of $0.93^{\circ}$ and $0.60^{\circ}$, respectively. A detailed description of the BrCEP instrument is presented in Yoon et al. ${ }^{27}$

BICEP observed two fields over the Galactic plane as shown in Figure 1. For each field, a telescope scans back-and-forth in azimuth at $2.8^{\circ} / \mathrm{s}$ over a $65^{\circ}$ range at a constant elevation. The elevation is stepped by $0.25^{\circ}$ after 50 right and left "half-scans" at a constant elevation. The telescope observed with four different orientations about its boresight: $0^{\circ}, 135^{\circ}, 180^{\circ}$, and $315^{\circ}$. Each observation of the single field has a fixed boresight angle and four observations cover all the boresight angles to increase the crosslinking coverage.

\subsection{Map making}

Figures 2 shows the $Q$ and $U$ maps observed by BrCEP at 100 and $150 \mathrm{GHz}$. This section describes polarized map making, focusing on processes unique to the Galactic field analysis. The map making process that is common to the CMB analysis is described in Chiang et al. ${ }^{8}$

The low-level time stream cleaning is applied to the raw time stream in following steps, (i) deconvolution of a bolometer transfer function, (ii) low-pass filtering at $5 \mathrm{~Hz}$, and (iii) downsampling to $10 \mathrm{~Hz}$. The $j^{\text {th }}$ sample in a half-scan of a gain adjusted pair-differenced time stream is

$$
d_{i}\left(t_{j}\right)=\frac{1}{2}\left(\frac{d_{i_{\mathrm{A}}}\left(t_{j}\right)}{g_{i_{\mathrm{A}}}}-\frac{d_{i_{\mathrm{B}}}\left(t_{j}\right)}{g_{i_{\mathrm{B}}}}\right),
$$



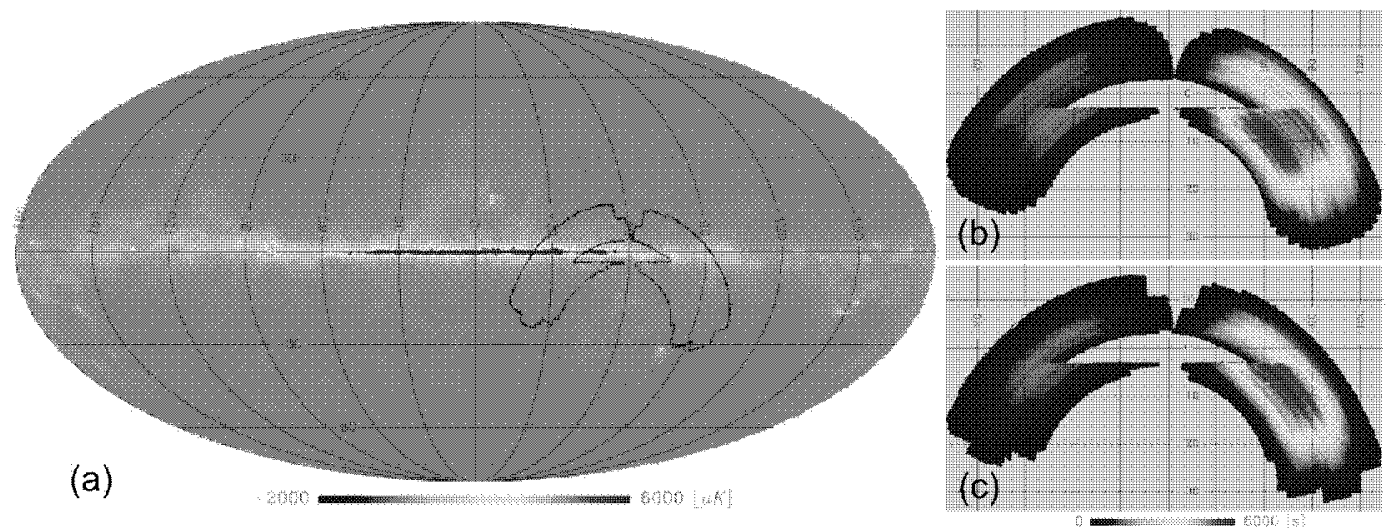

Figure 1. (a) The two BICEP Galactic regions are indicated over the FDS model 8 at $150 \mathrm{GHz}{ }^{28}$ Right: The integration time per nside $=256$ of Healpix pixel at $100 \mathrm{GHz}(\mathrm{b})$ and $150 \mathrm{GHz}$ (c) ${ }^{29}$
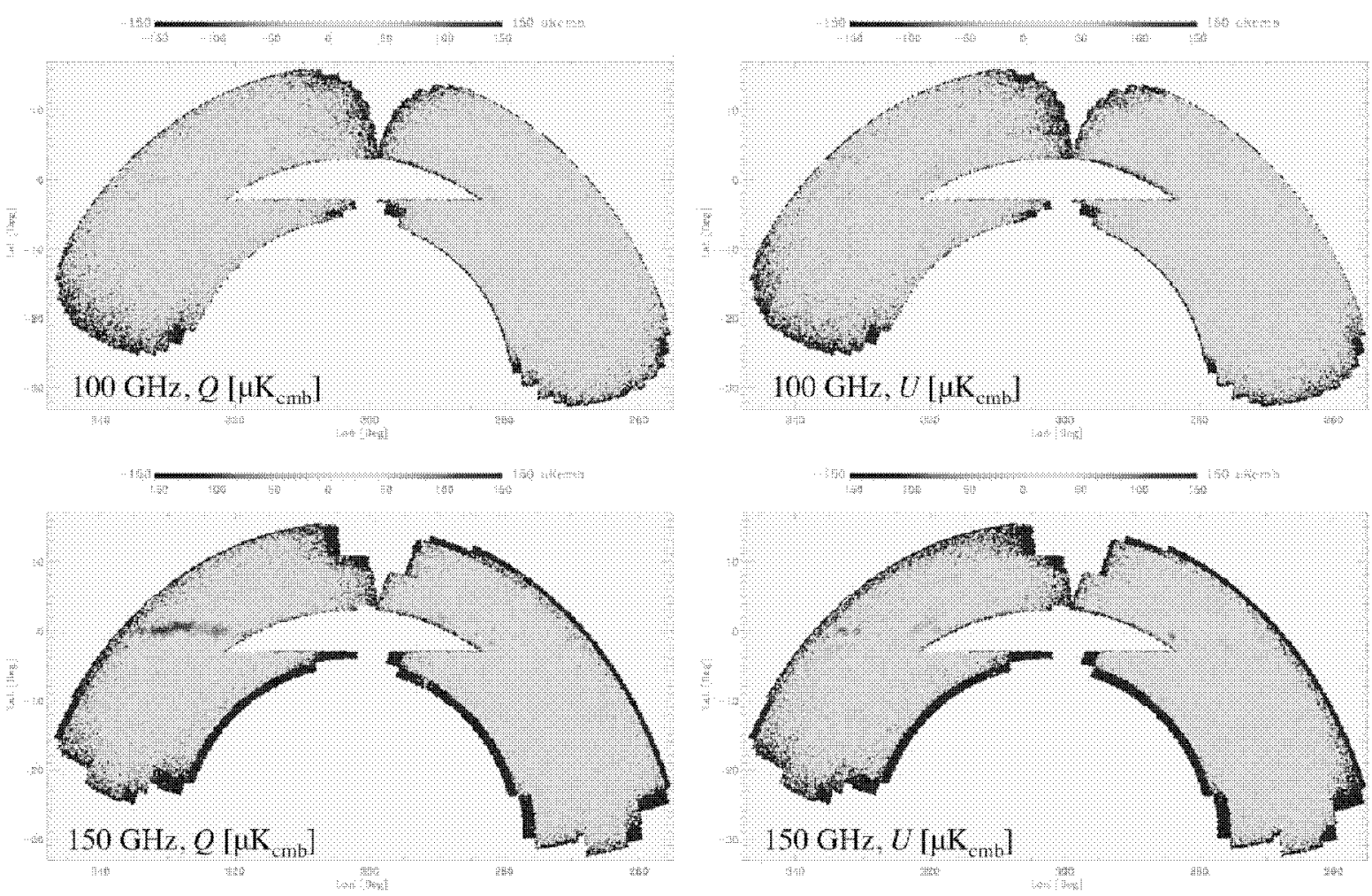

Figure 2. The $Q$ and $U$ maps of the Galactic fields are shown in the unit of $\mu K_{c m b} . Q$ and $U$ are defined in the Galactic coordinate with IAU convention.

where $d_{i_{\mathrm{A}, \mathrm{B}}}$ are the individual low-level processed time streams of $i^{\text {th }}$ PSB pair, and $g_{\mathrm{i}_{\mathrm{A}, \mathrm{B}}}$ are the gain factors for each PSB calibrated at every elevation step. Common mode noise between the PSB pair, such as thermal fluctuations of the instrument and atmospheric fluctuations, is removed by differencing between the two PSB pair time streams, and we fit a third order polynomial, $F_{i}\left(t_{j}\right)$, to the pair-differenced time stream in order to remove residual $1 / f$ noise below $\sim 0.1 \mathrm{~Hz}$.

When the telescope sweeps over the Galactic plane the variations in the time stream due to the Galactic signal and $1 / f$ noise are degenerate. To prevent removing the Galactic signal we apply a mask within the Galactic latitude of $\pm 3^{\circ}$. The mask is applied in the time domain and we only fit the polynomial to the pair-differenced time stream where the mask is not applied. The fitted polynomial is subtracted from the time stream $d_{i j}$ for all 
the samples in the half-scan.

In some cases one end of the half-scan lies inside of the Galactic mask. We did not include such half-scan in the map making because the polynomial inside of the mask needs to be extrapolated from the edge of the mask and the extrapolated polynomial does not represent the $1 / f$ noise inside of the mask. The half-scans whose two ends lie in the mask are also excluded. Consequently, the recovered maps become "pac-man" shaped. The recovered region shrinks as the mask width increases.

We follow the formalism described by Jones et al. ${ }^{30}$ and used for the BICEP CMB analysis described in Chiang et al. ${ }^{8}$ The $Q$ and $U$ values of the pixel in the direction on the sky $\vec{p}$ are computed from $d_{i j}^{\prime}=d_{i}\left(t_{j}\right)-F_{i}\left(t_{j}\right)$ as

$$
\left[\begin{array}{l}
Q(\vec{p}) \\
U(\vec{p})
\end{array}\right]=M \sum_{i}^{N_{P S B}} \sum_{j \subset \vec{p}} w_{i j}\left[\begin{array}{l}
d_{i j}^{\prime} \alpha \\
d_{i j}^{\prime} \beta
\end{array}\right],
$$

where

$$
M^{-1}=\frac{1}{2} \sum_{i}^{N_{P S B}} \sum_{j \subset \vec{p}} w_{i j}\left(\begin{array}{cc}
\alpha_{i j}^{2} & \alpha_{i j} \beta_{i j} \\
\alpha_{i j} \beta_{i j} & \beta_{i j}^{2}
\end{array}\right)
$$

and

$$
\begin{aligned}
& \alpha_{i j}=\gamma_{i_{\mathrm{A}}} \cos 2 \psi_{i_{\mathrm{A}} j}-\gamma_{i_{\mathrm{B}}} \cos 2 \psi_{i_{\mathrm{B}} j} \\
& \beta_{i j}=\gamma_{i_{\mathrm{A}}} \sin 2 \psi_{i_{\mathrm{A}} j}-\gamma_{i_{\mathrm{B}}} \sin 2 \psi_{i_{\mathrm{B}} j} .
\end{aligned}
$$

The weight $w_{i j}$ is an inverse of a variance of $d_{i j}^{\prime}$ calculated from the samples outside of the mask in each half-scan. The angle $\psi$ is the PSB orientations projected on the sky and $\gamma=\frac{1-\epsilon}{1+\epsilon}$ is the polarization efficiency factor, where $\epsilon$ is a cross-polarization response. Subscripts $i_{\mathrm{A}}$ and $i_{\mathrm{B}}$ refers to the $A$ and $B$ bolometers of the $i$ th pair. We use nside $=256$ of Healpix pixelization to project $Q$ and $U$ on the sky. ${ }^{29}$

\subsection{Pixel noise in the map}

Statistical errors in the BICEP maps are estimated using jackknife maps. We split the data into three pairs of halves, (i) right and left half-scans, (ii) $\left(0^{\circ}, 315^{\circ}\right)$ and $\left(135^{\circ}, 180^{\circ}\right)$ boresight angles, (iii) two sets of detector pairs located in alternating sectors of the 6-sector circular focal plane. We compute the $Q$ and $U$ maps of each data set as $\left(m_{Q_{1}}, m_{U_{1}}\right)$ and $\left(m_{Q_{2}}, m_{U_{2}}\right)$. We compute the difference as

$$
\delta m_{Q}=\frac{1}{2}\left(m_{Q_{1}}-m_{Q_{2}}\right) \sqrt{N}, \quad \delta m_{U}=\frac{1}{2}\left(m_{U_{1}}-m_{U_{2}}\right) \sqrt{N}
$$

where $N$ is the number of observations at each Healpix pixel. We compute the histogram of $N E Q=\delta m_{Q} / \sqrt{2 f_{s}}$ and $N E U=\delta m_{U} / \sqrt{2 f_{s}}$ (where $f_{s}=10 \mathrm{~Hz}$ sampling rate) from the map pixels that meet the criteria of (1) $N>2000$, (2) the Galactic latitude $\left|\theta_{\text {glat }}\right|<3^{\circ}$ and (3) not being at the edge of the observed regions. (Hereafter we call the region of the sky that meets these criteria a selected region.) We fit the histogram with a Gaussian $A \exp -\frac{(\delta m-\bar{m})^{2}}{2 \sigma_{0}^{2}}$.

Figure 3 shows the histograms of the jackknife maps. The noise property is well described by the Gaussian distribution. The averaged $N E Q$ and $N E U$ from the three jackknives are 523 and $507 \mu \mathrm{K} \sqrt{s}$ for $100 \mathrm{GHz}$, and 428 and $431 \mu \mathrm{K} \sqrt{s}$ for $150 \mathrm{GHz}$, respectively. Among the three jackknives the worst $N E Q$ is $16 \%$ larger than the best one that is from the right and left half-scan jackknife.

\subsection{Systematic error}

The polarization properties of a polarimeter are described by two quantities, PSB orientation $\psi$ and the crosspolarization response $\epsilon$. The PSB orientation $\psi$ is the angle at which the PSB is sensitive to the linear polarization. The cross-polarization response is the response of the PSB to the orthogonally polarized incident radiation. 

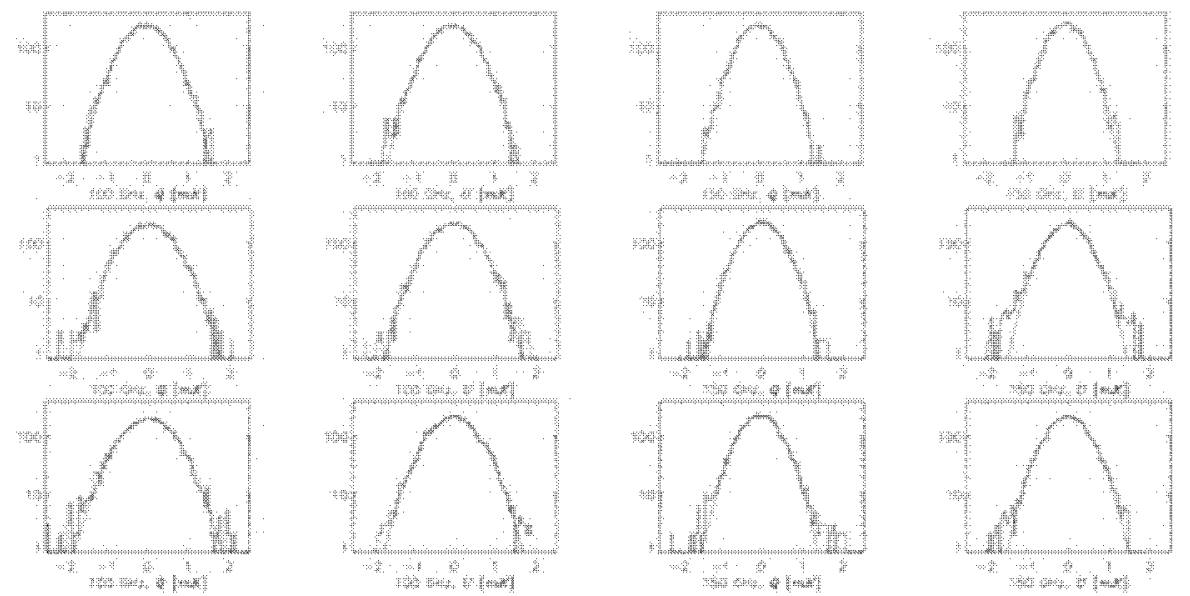

Figure 3. The histograms of $\delta m_{Q}$ and $\delta m_{U}$ for 100 and $150 \mathrm{GHz}$ are shown for right/left (top), boresight angle (middle), and detector sets (bottom) jackknives.
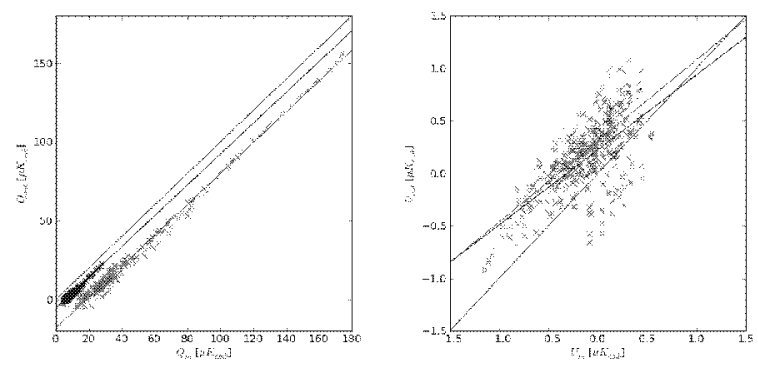

Figure 4. Comparison of the filtered $Q$ (and $U$ ) values of the simulated map against to the input unfiltered $Q$ (and $U$ ) values. The two linear lines are fits to the $100 \mathrm{GHz}$ (blue) and $150 \mathrm{GHz}$ (green) data. The red line indicates the line that has a slope of 1 with zero offset. The offsets of 100 and $150 \mathrm{GHz} Q$ signals are -5.6 and -18.1 , respectively.
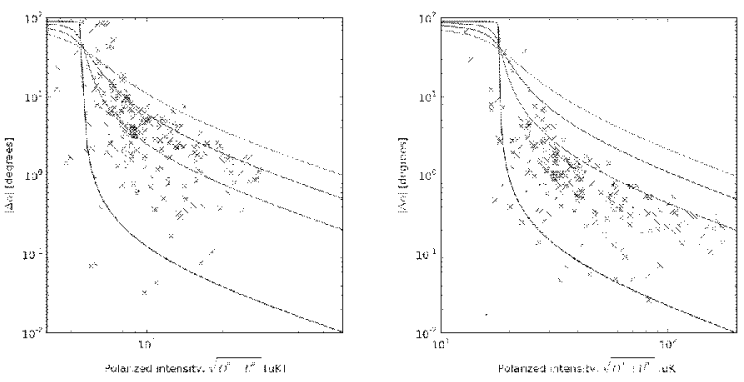

Figure 5. The difference of the polarization angle at each pixel before and after applying the BICEP time domain filtering to the simulated maps is plotted. The points are selected from the pixels for the Galactic latitude of $|\theta|<1$ (red), $|\theta|<2$ and $|\theta|>1$ (black), $|\theta|<3$ and $|\theta|>2$ (blue). The four lines are $|\Delta \alpha|$ from Equation 9 with the cases for $\alpha_{i n}$ of $0.1^{\circ}, 2^{\circ}, 5^{\circ}$ and $10^{\circ}$.

Two calibration methods are used to measure the cross-polarization. One uses a modulated linearly polarized broadband noise source mounted $200 \mathrm{~m}$ away from the BICEP telescope. BICEP observed the source by raster scans with 18 different boresight rotation angles. The other method uses a rotating wire grid mounted at the cryostat window. The signal is generated by chopping between an ambient absorber and the sky. The crosspolarization response is measured to within \pm 0.01 .

The PSB orientation is measured with a rotating dielectric sheet mounted in front of the cryostat window in addition to the two methods described to measure the cross-polarization. The measurements were repeated through each observing year and the uncertainty of the individual PSB orientation is $0.1^{\circ} \mathrm{rms}$. After the cryostat was opened between 2006 and 2007 observing years, the PSB orientation measurements showed an average of $1^{\circ}$ rotation in the absolute polarization angle. Thus, the absolute PSB orientation uncertainty is assigned to be less than $0.7^{\circ} \mathrm{rms}$ for three years of the observation periods. The detailed discussion of the polarization calibration of the BrCEP polarimeter is described in Takahashi et al. ${ }^{31}$

\subsection{Effects due to time domain filtering}

The subtraction of a polynomial fit from the pair-differenced time stream effectively acts as a high-pass filter in the time domain. While the purpose of the high-pass filtering is to remove $1 / f$ noise, this filtering also removes some modes of the signal. 
In order to quantify the amount of the time domain filtering of the Galactic signal, we prepare a simulated polarization map (Healpix pixelization of nside=256) that consists of the sum of CMB and FDS maps at 100 and $150 \mathrm{GHz}$ with a beam size of $0.93^{\circ}$ and $0.60^{\circ}$, respectively. ${ }^{28}$ The CMB map is generated by synfast using the cosmological parameters of the standard $\Lambda$ CDM model presented in Komatsu et al. ${ }^{20,29}$ The $Q$ polarization of the FDS map is made based on the relationships of $Q / T=c_{0}\left(T / T_{\max }\right)^{c_{1}}$ observed by BrCEP. ${ }^{32}$ We have used $\left(c_{0}, c_{1}\right)=(0.007,-0.47)$ and $(0.017,-0.29)$ for 100 and $150 \mathrm{GHz}$, respectively.

According to this polarization model, the Galactic $Q$ depends on temperature signal. The FDS model 8 does not have the same level of the emission as it is observed by the BICEP. In order to simulate the realistic level of the Galactic emission we use the temperature ' $T=\beta T_{F D S}$, where $\beta=1.30$ and 0.87 for 100 and $150 \mathrm{GHz}$, respectively. The $U$ polarization of the FDS is set to be zero for all the pixels. The simulated maps are smoothed to the beam size of $0.93^{\circ}$ and $0.60^{\circ}$ for 100 and $150 \mathrm{GHz}$, respectively.

We generate time ordered data using these simulated maps with the BICEP pointing and apply the same map making as we apply to the real data. Figure 4 shows the correlation between the input and filtered $Q$ and $U$ for the pixels inside of the selected sky region. The relationship of $Q$ before and after applying the time domain filtering is well described by a simple linear relationship. The offset generally depends on the amount of the signal contained at the Galactic plane and the offset is higher when the Galactic signal is higher. This is because the signal level at the mask boundary is significant as compared to the $1 / f$ noise, and therefore the interpolated polynomial inside of the mask follows the trend of the Galactic signal instead of the trend from the $1 / f$ noise. On the other hand, the $U$ polarization does not show any clear trend. This is because $U$ polarization do not contain any Galactic signal but only the polarization of the CMB. Therefore, there is no characteristic signal increase at the Galactic plane.

Figure 5 shows the change of the polarization angle after time domain filtering. The change of the polarization angle $|\Delta \alpha|$ is modeled as

$$
\begin{aligned}
Q_{f i l t} & =Q_{i n}-Q_{0} \\
U_{f i l t} & =U_{i n} \\
\Delta \alpha & =\frac{1}{2}\left(\arctan \frac{U_{f i l t}}{Q_{f i l t}}-\arctan \frac{U_{i n}}{Q_{i n}}\right) \\
& =\frac{1}{2}\left(\arctan \frac{I_{p} \sin 2 \alpha_{i n}}{I_{p} \cos 2 \alpha_{i n}-Q_{0}}-\alpha_{i n}\right)
\end{aligned}
$$

where $Q_{i n}=I_{p} \cos 2 \alpha_{i n}, U_{i n}=I_{p} \sin 2 \alpha_{i n}$ and $I_{p}=\sqrt{Q_{i n}^{2}+U_{i n}^{2}} . Q_{0}$ is the offset to account for the filtering effect. The time domain filtering effect to the polarization angle ranges from 0.1 to 100 degrees. Therefore, when the offset angle between the BICEP map and the map from other experiment is cross-calibrated it is important to apply the same time domain filtering to the other map.

\section{ESTIMATION OF THE OFFSET ANGLE AND ITS ERROR}

We describe a method to detect the overall polarization angle offset between the two polarization maps. We have two sets of $Q$ and $U$ maps. Ones are the BICEP maps as the calibrated maps. The others are maps to be calibrated. In the case of comparing the maps from two different experiments, they do not necessarily have the same beam sizes, and therefore we need to deconvolve the original beam and smooth the two maps to the same beam size. The choice of the beam smoothing varies depending on the beam sizes of BICEP and other experiment to be calibrated. In this section we assume that the two sets of maps have a same beam size and are pixelized such that the noise among pixels are not correlated. We discuss the treatment of the different beam size between the separate experiments as a case-by-case basis in Section 4.

We write the second and third components of the Stokes parameter of $i$ th pixel in the two sets of maps as

$$
\begin{array}{rll}
\text { BrCEP map }: & \left(Q_{i B} \pm \delta Q_{i B}, U_{i B} \pm \delta U_{i B}\right), \\
\text { Uncalibrated map }: \quad & \left(Q_{i} \pm \delta Q_{i}, U_{i} \pm \delta U_{i}\right)
\end{array}
$$

where $\delta Q$ and $\delta U$ indicate the statistical noise. We assume that the parent distribution of the pixel noise is a Gaussian described by the standard deviation of $\sigma_{Q_{i(B)}}, \sigma_{U_{i(B)}}, \sigma_{Q U_{i(B)}}$ with a mean of zero. 
We relate $Q$ and $U$ of the same pixel on the sky between two experiments by two parameters, offset angle $\delta \alpha$ and the ratio of the polarized amplitudes $\rho$ as

$$
\left[\begin{array}{c}
Q_{i} \\
U_{i}
\end{array}\right]=\rho_{i}\left[\begin{array}{cc}
\cos 2 \delta \alpha_{i} & \sin 2 \delta \alpha_{i} \\
-\sin 2 \delta \alpha_{i} & \cos 2 \delta \alpha_{i}
\end{array}\right]\left[\begin{array}{c}
Q_{B i} \\
U_{B i}
\end{array}\right]
$$

We can solve Equation 12 for $\rho_{i}$ and $\alpha_{i}$ as,

$$
\left[\begin{array}{c}
\rho_{i} \cos 2 \delta \alpha_{i} \\
\rho_{i} \sin 2 \delta \alpha_{i}
\end{array}\right]=\frac{1}{Q_{B i}^{2}+U_{B i}^{2}}\left[\begin{array}{cc}
Q_{B i} & U_{B i} \\
U_{B i} & -Q_{B i}
\end{array}\right]\left[\begin{array}{c}
Q_{i} \\
U_{i}
\end{array}\right]
$$

and thus

$$
\begin{aligned}
\delta \alpha_{i} & =\frac{1}{2} \arctan \frac{U_{B i} Q_{i}-Q_{B i} U_{i}}{Q_{B i} Q_{i}+U_{B i} U_{i}} \\
& =\alpha_{B i}-\alpha_{i}, \\
\rho_{i} & =\sqrt{\frac{Q_{i}^{2}+U_{i}^{2}}{Q_{B i}^{2}+U_{B i}^{2}}},
\end{aligned}
$$

where $\alpha_{B i}=\frac{1}{2} \arctan U_{B i} / Q_{B i}$ and $\alpha_{i}=\frac{1}{2} \arctan U_{i} / Q_{i}$. When the $Q$ and $U$ maps from two separate experiments are identical, $\alpha_{i}=0$ and $\rho_{i}=1$.

While the polarization calibration can be done in terms of $Q$ and $U$, we express $Q$ and $U$ of two maps in terms of $\delta \alpha$ and $\rho$. This choice was made to mitigate the effect due to the spectral dependence of the instrumental bandpass location and shape mismatch between the two separate experiments. We discuss the spectral dependence of the polarization angle in Section 5.

When the $Q$ and $U$ maps contain only signals, we have a perfect knowledge of the offset angle $\delta \alpha$ for every pixel. When the noise is present in the maps, the noise in the map has to be propagated to an error in the offset angle. The error of the offset angle in each pixel $i$ is

$$
\sigma_{\delta \alpha_{i}}^{2} \simeq\left(\frac{\partial \delta \alpha_{i}}{\partial Q_{B i}}\right)^{2} \sigma_{Q_{B i}}^{2}+\left(\frac{\partial \delta \alpha_{i}}{\partial U_{B i}}\right)^{2} \sigma_{U_{B i}}^{2}+\frac{\partial \delta \alpha_{i}}{\partial Q_{B i}} \frac{\partial \delta \alpha_{i}}{\partial U_{B i}} \sigma_{Q U_{B i}}^{2}+\left(\frac{\partial \delta \alpha_{i}}{\partial Q_{i}}\right)^{2} \sigma_{Q_{i}}^{2}+\left(\frac{\partial \delta \alpha_{i}}{\partial U_{i}}\right)^{2} \sigma_{U_{i}}^{2}+\frac{\partial \delta \alpha_{i}}{\partial Q_{i}} \frac{\partial \delta \alpha_{i}}{\partial U_{i}} \sigma_{Q U_{i}}^{2},
$$

where $\sigma_{Q_{B i}}, \sigma_{U_{B i}}, \sigma_{Q U_{B i}}, \sigma_{Q_{i}}, \sigma_{U_{i}}, \sigma_{Q U_{i}}$ are the pixel noise associated with $Q_{B i}$ and $U_{B i}$, and $Q_{i}$ and $U_{i}$, respectively. The derivative terms are

$$
\begin{array}{rlrl}
\frac{\partial \delta \alpha_{i}}{\partial Q_{B i}}=-\frac{1}{2} \frac{U_{B i}}{Q_{B i}^{2}+U_{B i}^{2}}, & & \frac{\partial \delta \alpha_{i}}{\partial U_{B i}}=\frac{1}{2} \frac{Q_{B i}}{Q_{B i}^{2}+U_{B i}^{2}} \\
\frac{\partial \delta \alpha_{i}}{\partial Q_{i}}=\frac{1}{2} \frac{U_{i}}{Q_{i}^{2}+U_{i}^{2}}, & \frac{\partial \delta \alpha}{\partial U_{i}}=-\frac{1}{2} \frac{Q_{i}}{Q_{i}^{2}+U_{i}^{2}} .
\end{array}
$$

The derivative terms are inversely proportional to the square of the polarized intensity. This indicates that the error of the offset angle is smaller when the polarized intensity is stronger. Figure 6 shows the angle uncertainty as a function of the pixel noise in $Q$ and $U$ maps and the polarized intensity, $\sqrt{Q^{2}+U^{2}}$.

While a polarization angle $\alpha_{i}$ varies from -90 to 90 degrees based on the signal and the pixel noise at the given point on the sky, we assume that the distribution of the differenced angle $\delta \alpha_{i}$ is a Gaussian. We validate this assumption in Section 4 when we apply this formalism between BICEP and WMAP.

The Galaxy is not a single point source, and therefore the estimation of the polarization offset angle improves by including all the available pixels in the map. In order to calculate the mean polarization offset angle, $\delta \alpha_{0}$, between the reference and the uncalibrated polarimeter maps and the corresponding uncertainty of the mean, we compute the likelihood of $\delta \alpha_{0}$ as

$$
L \propto \mathrm{e}^{-\frac{1}{2} \chi^{2}\left(\delta \alpha_{0}\right)}
$$

where

$$
\chi^{2}\left(\delta \alpha_{0}\right)=\sum_{i} \frac{\left(\delta \alpha_{i}-\delta \alpha_{0}\right)^{2}}{\sigma_{\delta \alpha_{i}}^{2}}
$$




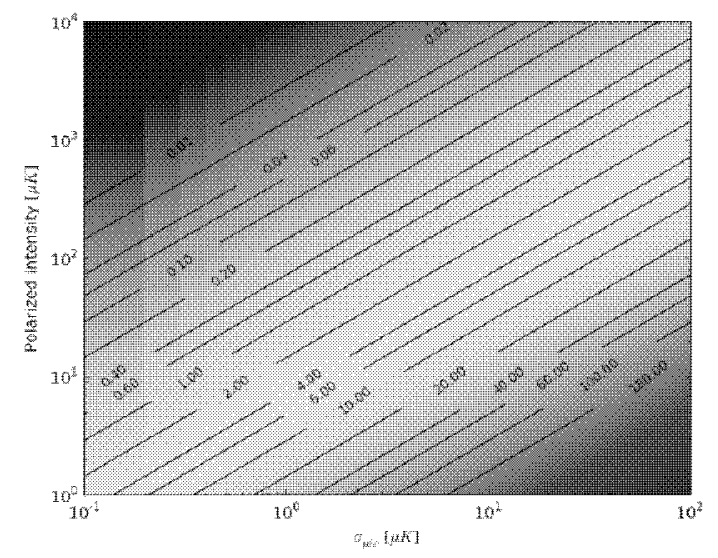

Figure 6. This plot shows the angle uncertainty of the polarization signal at a given pixel as a function of a pixel noise and a polarized intensity of the signal. For an example, a map that has a pixel noise of $10 \mu K$ and polarized intensity of $100 \mu K$ has a polarization angle error of $3^{\circ}$.

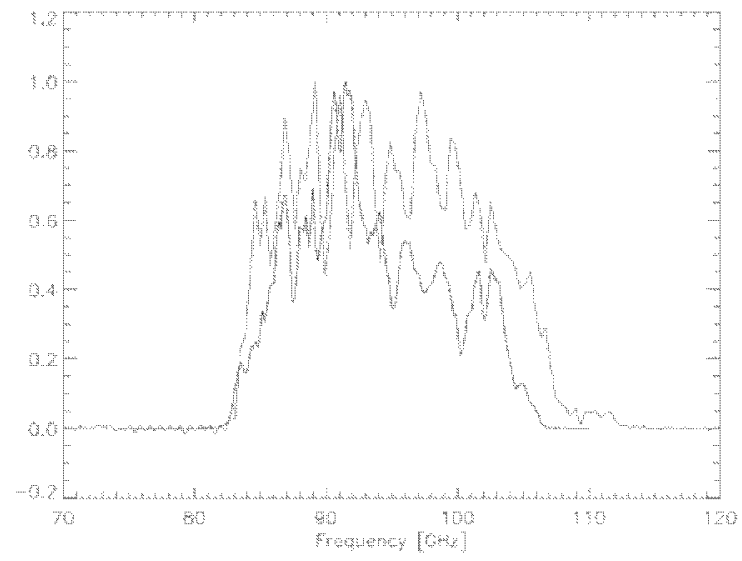

Figure 7. The spectra of BICEP (red) and WMAP $W$ band (black). Both spectra are normalized to the maximum value of 1 . The BICEP spectrum is the average of a PSB pair. The WMAP spectrum is the average of the $W$ band spectra.

\section{RESULTS}

We apply the method described in the previous section to BICEP and WMAP. We also compute the expected constraint to Planck and EPIC, by using BICEP Galactic map.

\subsection{Polarization angle offset between BICEP and WMAP}

WMAP has been observing the temperature and polarization over the full sky. ${ }^{33}$ The spectral bandwidth of BICEP $100 \mathrm{GHz}$ and the WMAP W band overlap as shown in Figure 7. In this exercise, we assume that the absolute polarization angle of the WMAP polarimeter is unknown and we constrain the overall offset angle of the WMAP polarization maps using the BrCEP Galactic map as a polarization calibration source.

Before we apply the formalism described in Section 3, we need to correct the beam size difference between the two experiments. The FWHM of the BICEP beam size at $100 \mathrm{GHz}$ is $0.93^{\circ}$. Each of the $Q$ and $U$ maps of the four WMAP $W$ band differencing assembly is deconvolved with the corresponding WMAP $B_{l}$ and convolved with FWHM of $0.93^{\circ}$ Gaussian beam in nside $=512$ pixelization. We compute the weighted averaged map from the four differencing assembly maps in $\mathrm{W}$ band. The weights are the inverse of the pixel variance of each differencing assembly. We apply the BrCEP time domain filtering to the averaged WMAP W band map. The filtered $Q$ and $U$ maps are downsampled to $0.92^{\circ}$ pixel size (nside-64) maps in order to decorrelate the noise among pixels. The BICEP $Q$ and $U$ maps are also downsampled to the same pixelization.

The pixel noise of the WMAP maps is computed by $\sigma=\frac{\sigma_{0}}{\sqrt{N_{\text {hits }}}}$ where $\sigma_{0}$ for the WMAP W band differencing assemblies is $\left(\sigma_{W_{1}}, \sigma_{W_{2}}, \sigma_{W_{3}}, \sigma_{W_{4}}\right)=(5.940,6.612,6.983,6.840) \mathrm{mK}$ with nside $=512$ pixelization. We neglect the correlated noise between $Q$ and $U$ for both experiments. The pixel noise of the BICEP maps is computed based on the $N E Q(U)$ derived from the right and left jackknife maps.

Once the two sets of the maps and weights are computed in the same pixelization, we impose the criteria to select the pixels. We choose the pixels that meet the criteria of $\left|\theta_{\text {glat }}\right|<3^{\circ}, N_{\text {hits }}$ of BICEP $>2000$ and pixels of which its neighbor do not have $N_{\text {hits }}=0$. The second criterion assures that the most of the edge pixels of the map are not included. The second criterion does not exclude the pixel around $282^{\circ}<\phi_{\text {glon }}<322^{\circ}$ and $\left|\theta_{\text {glat }}\right|<3^{\circ}$ where the edge of the map is not tapered by $N_{\text {hits }}$. Therefore, we include the third criterion to exclude all the edge pixels in the maps.

Figure 8 shows the map of offset angle $\delta \alpha_{i}$ and the weight $1 / \sigma_{\delta \alpha_{i}}^{2}$. It is clear that the the offset angle is close to zero and the weight is higher at the Galactic plane. Figure 9 shows the weighted histogram of $\delta \alpha_{i}$. The 

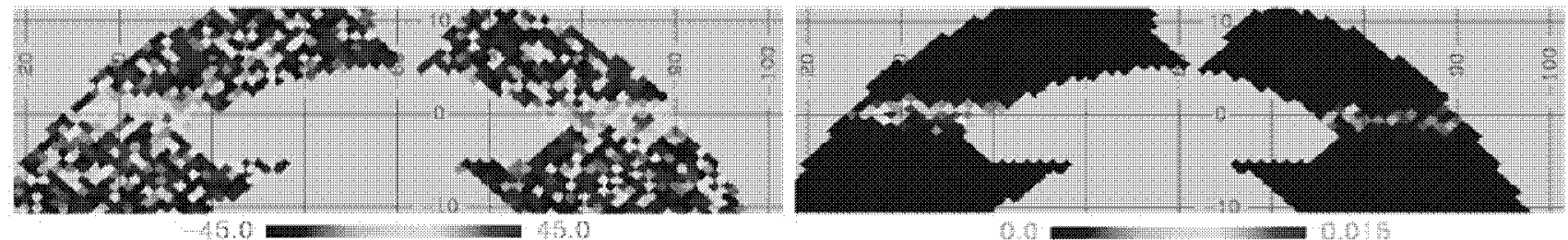

Figure 8. The maps of $\delta \alpha_{i}$ (left) in unit of degrees and weight $=1 / \sigma_{\delta \alpha_{i}}^{2}$ (right) in unit of degree ${ }^{-2}$. The maps are downsampled to Healpix resolution of nside $=64$. The edge pixels are removed, and therefore the shape of the map does not coincide with the ones in Figure 2.

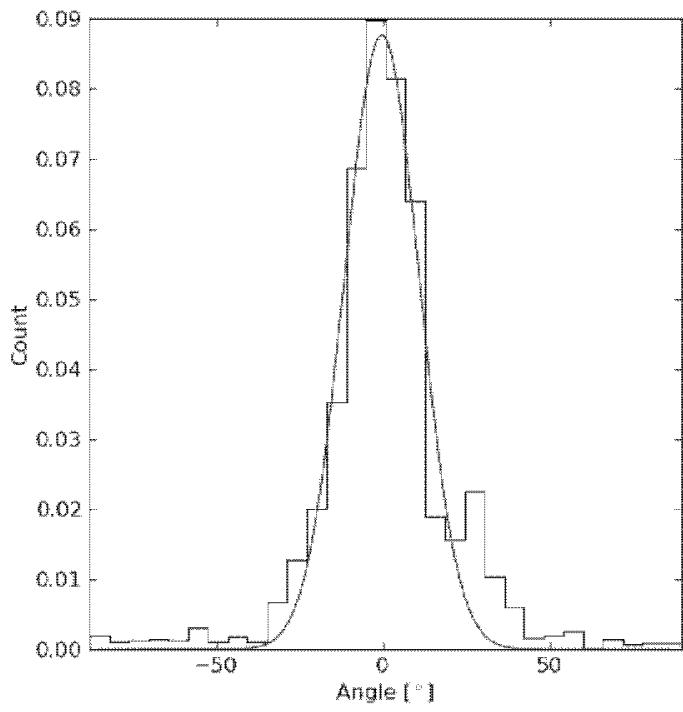

Figure 9. The weighted histogram of $\delta \alpha$ between the polarization maps of BICEP and WMAP and the Gaussian fit are shown. The distribution is well described as a Gaussian.

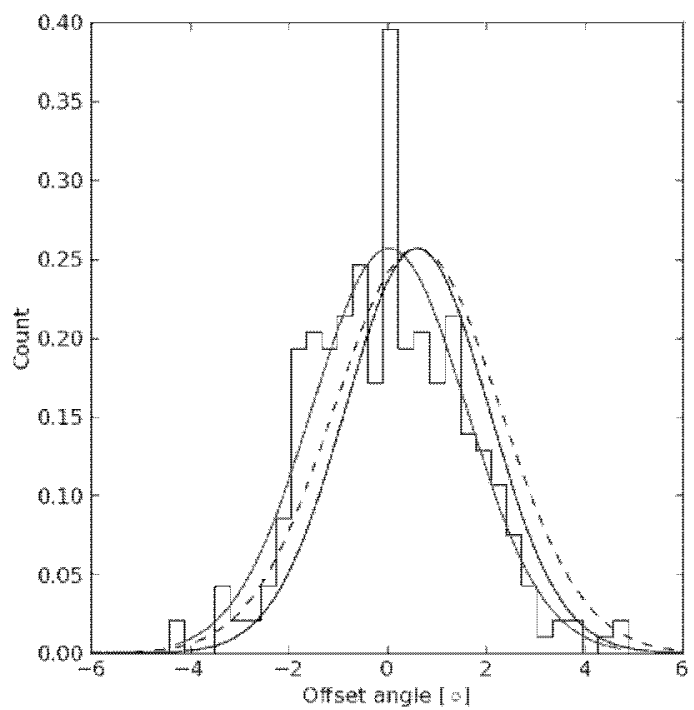

Figure 10. The likelihood of $\delta \alpha$ (solid black) with the pixel noise estimated from the right and left jackknife and $\delta \alpha$ (dash black) with the pixel noise increased by $16 \%$ as a worst pixel noise estimation. The histogram is a mean of the Gaussian fit to $\delta \alpha$ from the two sets of the simulated signal (CMB+FDS) at $100 \mathrm{GHz}$ and the 300 noise realizations. The red curve is the Gaussian fit to the histogram.

mean and the standard deviation of the angle uncertainty of each pixel is $-0.41^{\circ}$ and $11.2^{\circ}$, respectively. The distribution of the histogram is well described as a Gaussian distribution.

Figure 10 shows the likelihood of the offset angle calculated based on Equation 19 using the BrCEP and the filtered WMaP maps. The black line shows that the mean and the sigma are the $0.6^{\circ}$ and $1.4^{\circ}$ respectively. The dashed line with the same mean has $16 \%$ larger sigma as a worst case pixel noise.

The histogram in Figure 10 is the results of the signal and noise simulations. We prepare two sets of maps by adding the white noise of the BrCEP $100 \mathrm{GHz}$ and WMAP W band to the simulated signal only maps at $100 \mathrm{GHz}$ described in Section 2.4. We repeat computing the mean of the Gaussian fit to the histogram of $\delta \alpha$ from the two sets of the map for the 300 noise realization. The fit to this histogram in Figure 7 is consistent with the likelihood obtained by the using Equation 19.

\subsection{Polarization offset angle between BICEP and future experiments}

Any ongoing and forthcoming CMB polarization experiments which observe the BrCEP Galaxy region can crosscalibrate their polarization angle using the BICEP map. As examples, we compute the expected angle constraint 


\begin{tabular}{c|cc}
\hline & \multicolumn{2}{|c}{$1 \sigma$ error $\left[{ }^{\circ}\right]$} \\
Reference $\times$ Uncalibrated & $100 \mathrm{GHz}$ & $150 \mathrm{GHz}$ \\
\hline \hline BICEP $\times$ No noise experiment & 1.24 & 0.27 \\
\hline BICEP $\times$ WMAP W-band & 1.45 & \\
\hline BICEP $\times$ Planck & 1.26 & 0.27 \\
BICEP $\times$ EPIC-IM $(4 \mathrm{~K}$ option) & 1.24 & 0.26 \\
\hline (BICEP, BICEP2) $\times$ Planck & 1.26 & 0.08 \\
(BICEP, BICEP2, KECK $\times$ Planck & 0.23 & 0.06
\end{tabular}

Table 1. The $1 \sigma$ statistical error of the polarization angle offset for various combinations of the experiments. The first rOw, BICEP $\times$ No noise experiment, indicates the angle error only due to the BICEP statistical noise. BICEP 2 only has $150 \mathrm{GHz}$ band, and therefore the error in $100 \mathrm{GHz}$ does not show any improvement.

for two cases, BrCEP and Planck, and BICEP and EPIC. ${ }^{35}$

Table 1 shows the list of $1 \sigma$ statistical error from the likelihood in Equation 19 for $100 \mathrm{GHz}$ and $150 \mathrm{GHz}$ for the two experiments. In this comparison, we assume that the bandpass shape of the two separate experiments is the same and the knowledge of the beam shape is perfect.

The expected pixel noise of Planck and EPIC-IM are from Planck bluebook and Bock et al., respectively. ${ }^{34,35}$ It is clear that the estimate of the $1 \sigma$ error of the offset angle between BICEP and WMAP improves with BICEP and Planck or EPIC-IM. This is because the noise contribution from Planck and EPIC-IM is much smaller than the case from WMAP while the BrCEP noise stays the same. On the other hand, there is negligible improvement from Planck to EPIC-IM because the source of the noise in these two cases is limited by the pixel noise of the BICEP map.

While the observations of BICEP were completed, the ongoing BICEP2 and forthcoming KECK will improve the sensitivity to the angle calibration. If we assume that BICEP2 and KECK will spend the same observational time with the same detector sensitivity on the BICEP Galactic field, the expected reduction of the pixel noise is simply scaled by $\sqrt{\frac{N_{\text {Bicep }}}{N_{\text {Bicep }}+N_{0}}}$, where $N_{\text {Bicep }}$ is the number of detectors of BICEP and $N_{0}$ is of BICEP2 or BICEP2 and KeCK. We assume that $N_{\text {Bicep }}$ is 25 and 24 for 100 and $150 \mathrm{GHz}$, and $N_{0}$ is 256 for BrCEP2 $150 \mathrm{GHz}$ and $144 \times 4$ and $256 \times 2$ for 100 and $150 \mathrm{GHz}$ of KECK, respectively. The data combining with BrCEP 2 and KECK provide the statistical errors of the offset angle smaller than the systematic errors of the BrceP polarimeter itself for both 100 and $150 \mathrm{GHz}$ bands.

\section{DISCUSSION}

\subsection{Comparison between the diffuse Galactic source and the Crab nebula as a polarized calibration source}

We compare the Crab nebula and the BICEP Galactic region as a polarized source. The emission mechanism of the Crab nebula at the millimeter wavelength is dominated by the synchrotron emission. Macias-Perez et al. and Weiland et al. reported that the observed flux has a power law of $\propto\left(\frac{\nu}{40 G H z}\right)^{-0.3 \sim-0.35}$ while the degree of polarization stays constant around $7 \%$ over the millimeter wavelength. ${ }^{24,36}$ On the other hand, the diffuse dust emission at the Galactic plane increases as a function of frequency. ${ }^{32}$ Therefore, the signal-to-noise increases as the bandpass location increases.

The Crab nebula is a point-like source and the Galaxy is a diffuse source. In order to compare the two sources, we compute the integrated polarized flux of the Galactic field as shown in Table 2. We also show the integrated polarized flux reported in Weiland et al. and Aumont et al. ${ }^{21,24}$ The spatial area of the Galactic field is much larger than that of the Crab nebula. Therefore, the integrated polarized flux of the Galactic signal within the BICEP field is larger than that of the Crab nebula. The total pixel noise from the Galactic field is added in quadrature. As a result, the polarized Galactic source at $150 \mathrm{GHz}$ provides the same order of error as compared to the Crab nebula at $\mathrm{W}$ band. 


\begin{tabular}{c|ccc}
\hline Band & $Q[\mathrm{Jy}]$ & $U[\mathrm{Jy}]$ & Ang]e [ $]$ \\
\hline \hline WMAP, Crab nebula & & & \\
K band & $-27.13 \pm 0.68$ & $-1.40 \pm 0.08$ & $-88.5 \pm 0.1$ \\
Ka band & $-23.72 \pm 0.45$ & $-1.88 \pm 0.12$ & $-87.7 \pm 0.1$ \\
Q band & $-22.03 \pm 0.60$ & $-2.06 \pm 0.14$ & $-87.3 \pm 0.2$ \\
V band & $-19.25 \pm 0.36$ & $-1.52 \pm 0.24$ & $-87.7 \pm 0.4$ \\
W band & $-16.58 \pm 0.73$ & $-0.75 \pm 0.42$ & $-88.7 \pm 0.7$ \\
all band combined & & & 0.07 \\
\hline Aumont et al., Crab nebula & & \\
90 GHz & & & $-88.8 \pm 0.2$ \\
\hline BICEP Galaxy & $114.7 \pm 6.0$ & $29.1 \pm 6.2$ & \pm 1.5 \\
100 GHz & $573.4 \pm 12.2$ & $195.6 \pm 11.2$ & \pm 0.5 \\
150 GHz &
\end{tabular}

Table 2. The integrated polarized flux of the Crab nebula and the BICEP Galactic region is shown. The polarization convention in this paper and WMAP are different, and thus the sign of $U$ is changed from the original WMAP paper. The angle error of the BICEP Galactic measurements is the quadrature sum of the pixel noise. ${ }^{\dagger}$ The polarization angle seen by $10^{\prime}$ beam from Aumont et al. The original literature quoted the polarization angle in equatorial coordinates as $\alpha=148.8^{\circ}$.

\subsection{Effect of spectral mismatch between two experiments}

When the two polarization maps from two separate experiments are cross-calibrated, the spectral bandpass of the two experiments is not necessary the same. We assess the effect of the bandpass mismatch to the offset angle estimation between the BICEP $100 \mathrm{GHz}$ band and the WMAP W band.

Gold et al. derived the synchrotron and dust emission templates by the Markov chain Monte Carlo fitting., ${ }^{37}$ We compute the simulated BICEP and WMAP maps by integrating the sum of the synchrotron and dust template maps over the BICEP $100 \mathrm{GHz}$ bandpass and WMAP W band bandpass. We compute the offset angle $\delta \alpha_{i}$ of each pixel between the two bandpass maps. The median offset angle of all the pixels within the selected sky region is $0.005^{\circ}$. We define the signal-to-noise for each pixel as the ratio of the polarized intensity to the pixel noise. We compute the median and the maximum offset angle of which the pixels are the signal-to-noise $>3$ are $0.01^{\circ}$ and $0.02^{\circ}$, respectively.

The BICEP $100 \mathrm{GHz}$ map expects a higher contribution of the dust emission as compared to the WMAP W band map because the BICEP $100 \mathrm{GHz}$ bandwidth is slightly wider than WMAP W band bandwidth in higher frequency side as shown in Figure 7. Gold et al. shows that in the BICEP Galactic field the polarization direction of the synchrotron emission is $-26^{\circ}<\alpha_{\text {synch }}<0^{\circ}$ and that of the dust emission is $\left|\alpha_{d u s t}\right|<0.3^{\circ}$. Therefore, the overall offset angle between the BICEP and WMAP maps is expected to show the positive rotation due to the bandpass mismatch. The overall offset angle between the BICEP and WMAP maps, shown in Figure 7 , is $0.6^{\circ} \pm 1.4^{\circ}$, and the positive mean value is consistent with the bandpass mismatch.

This effect is prominent when the passband of the instrument is located where more than two emission spectra are mixed with nearly the same amplitude. This is because the two sources with different spectral shape can have different polarization angles.

\section{CONCLUSION}

We present the polarized diffuse Galactic emissions observed by BrCEP at 100 and $150 \mathrm{GHz}$ and the method to cross-calibrate the absolute angle between the BICEP map and any uncalibrated map. The absolute angle of the BICEP polarimeter is calibrated to $\pm 0.7^{\circ}$ and the $1 \sigma$ error of the polarization angle due to the pixel noise of the BICEP map is $1.24^{\circ}$ and $0.27^{\circ}$ for 100 and $150 \mathrm{GHz}$, respectively.

We apply this method between the BICEP and WMAP W band maps and cross-calibrate the angle to $0.6 \pm 1.4^{\circ}$. The expected $1 \sigma$ errors for the Planck 100 and $150 \mathrm{GHz}$ bands are $1.26^{\circ}$ and $0.27^{\circ}$, respectively. The ongoing and forthcoming BICEP2 and KECK are expected to reduce the statistical noise of the observations of the BICEP Galactic region significantly. 
The BICEP Galactic maps provide the polarized Galactic emission as a new angle calibration source for the ongoing and forthcoming CMB B-mode experiments that require the absolute angle calibration to a fraction of a degree. The method of using the Galactic signal as an angle calibration source can be applied to any two experiments if one of the polarimeters is well calibrated. Therefore, when the Planck full sky polarization maps are available, the future polarimeters should be able to use the Galactic signal as a calibration source not only with respect to BrCEP but also to Planck.

\section{REFERENCES}

[1] W. Hu and M. White, A CMB polarization primer, New Astronomy, 2, pp. 323-344, 1997.

[2] J. M. Kovac 2002, Detection of polarization in the cosmic microwave background using DASI, Nature, 420, 772 , astro-ph/0209478.

[3] T. Montroy et al. 2006 ApJ 647813.

[4] Sievers et al., The Astrophysical Journal, 660:976 987, 2007 May 10.

[5] J. H. P. Wu et al. 2007 ApJ 66555.

[6] M. L. Brown et al. 2009 ApJ 705978.

[7] L. Page, et al., 2007, ApJS, 170, 335.

[8] H. C. Chiang et al. 2010 ApJ 7111123.

[9] L. Pagano et al., Phys. Rev. D80:043522,2009.

[10] Reichborn-Kjennerud et al. Submitted to these proceedings.

[11] Ogburn et al., Submitted to these proceedings.

[12] Sheehy et al., Submitted to these proceedings.

[13] Arnold et al., Submitted to these proceedings.

[14] D. Samtleben et al., In the Proceedings 'A Century of Cosmology', San Servolo (Venezia, Italy), August 2007. arXiv:astro-ph/0802.2657.

[15] Crill et al. Proceedings of SPIE Volume 7010. arXiv:0807.1548v1.

[16] Filippini et al. Submitted to these proceedings.

[17] Weiss et al. Task Force on Cosmic Microwave Background Research,' arXiv:astro-ph/0604101v1.

[18] J. Q. Xia et al., Phys. Lett. B687:129-132,2010.

[19] Wi1 et al. QUaD Collaboration. Phys. Rev. Lett. 102, 161302 (2009).

[20] Komatsu et al. Seven-Year Wilkinson Microwave Anisotropy Probe (WMAP) Observations. Submitted to ApJ. http://lambda.gsfc.nasa.gov/

[21] Aumont et al. A\&A 514, A70 (2010).

[22] Zemcov et al. 2010 ApJ. 7101541.

[23] Leitch et al., Nature 420 (2002) 763-771.

[24] Weiland et al. Seven-Year Wilkinson Microwave Anisotropy Probe (WMAP) Observations. Submitted to ApJ. http://lambda.gsfc.nasa.gov/

[25] Tauber et al., The Extragalactic Infrared Background and its Cosmological Implications. IAU Symposium, vol. 2042001.

[26] Rosset, C. et al., "'" Accepted by Astronomy\&Astrophysics, April 12, (2010).

[27] Yoon, K. W., et al. 2006, Proceedings of SPIE Volume 6275,

[28] Finkbeiner, D. P., Davis, M., \& Schlegel, D. J. 1999, Astrophys. J. , 524, 867, astro-ph/9905128.

[29] Gorski, K.M. et al., 2005, Astrophys. J., 622,759.

[30] W. C. Jones et al., Astr. \& Astroph. , 470, Issue 2, 771785, August 2007, astro-ph/0606606.

[31] Y. D. Takahashi et al. 2010 ApJ 7111141.

[32] Bierman et al. Submitted to these proceedings.

[33] Jarosik et al. Seven-Year Wilkinson Microwave Anisotropy Probe (WMAP) Observations. Submitted to ApJ. http://lambda.gsfc.nasa.gov/

[34] Planck bluebook. ESA-SCI(2005)1. http://www.rssd.esa.int/Planck/

[35] J. Bock et al. Study of the Experimental Probe of Inflationary Cosmology (EPIC)-Intemediate Mission for NASA's Einstein Inflation Probe. arXiv:0906.1188v1.

[36] Marcias-Perez et al., ApJ. 711:417-423, 2010 March 1.

[37] Gold et al. Seven-Year Wilkinson Microwave Anisotropy Probe (WMAP) Observations. Submitted to ApJ. http://lambda.gsfc.nasa.gov/ 